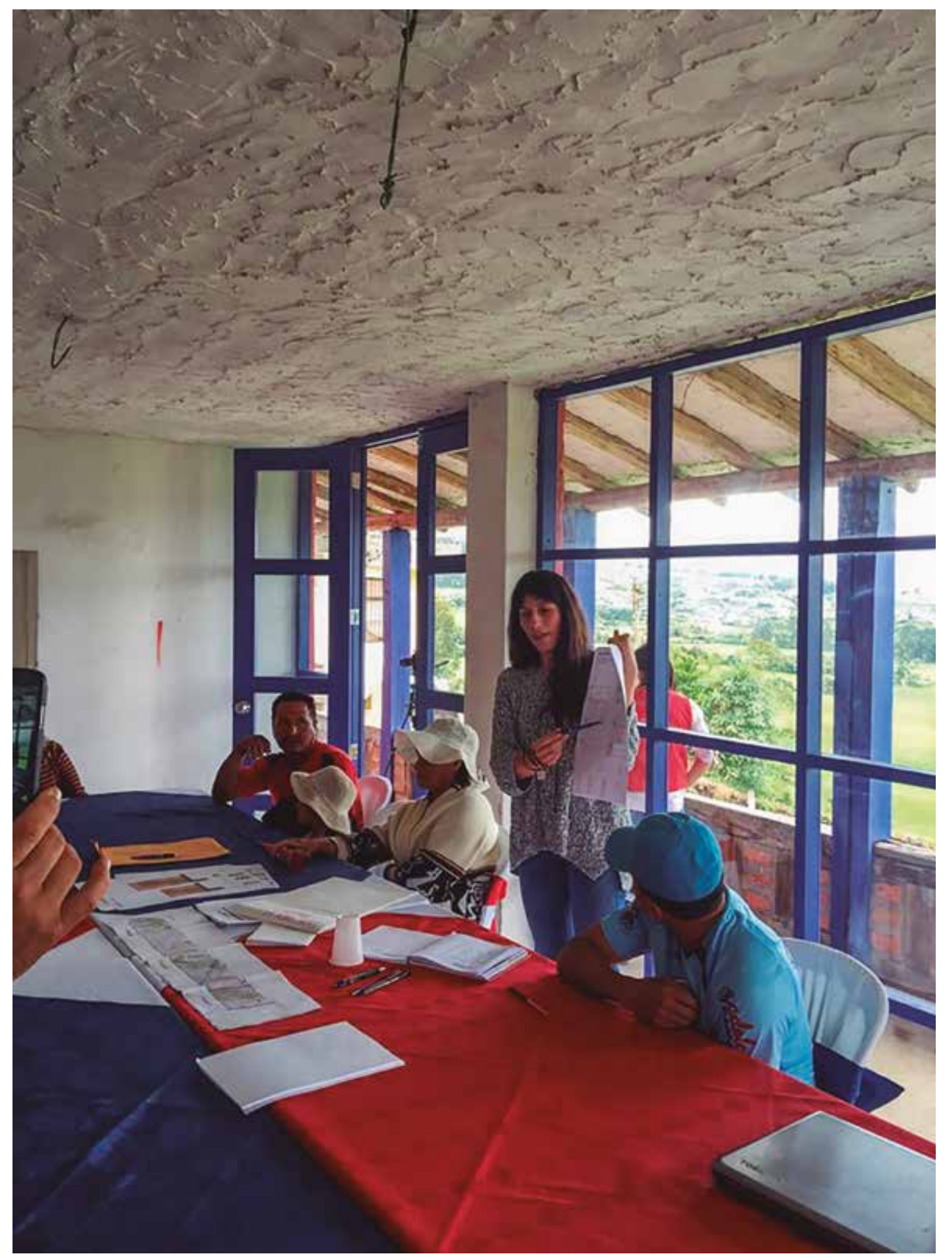

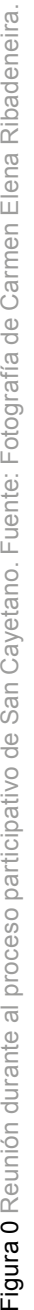



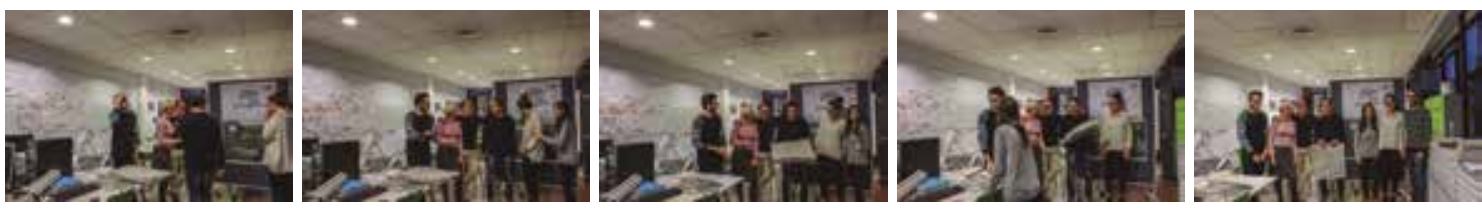

Erika Raquel Ramos, Daniel Crisóstomo, Antonio Díaz Jerez y Germán Colomer

Fotos: Germán Colomer

\title{
UNA EXPERIENCIA DE PROCESO PARTICIPATIVO PROMOVIDA POR LA EMPRESA PRIVADA EN EL CONTEXTO DE LA CONSTRUCCIÓN DE NUEVOS BARRIOS SUSTENTABLES: EL CASO DE SAN CAYETANO EN MEJÍA, ÁREA METROPOLITANA DE QUITO, ECUADOR'
}

\section{A PARTICIPATORY PROCESS EXPERIENCE PROMOTED BY PRIVATE ENTERPRISE IN THE CONTEXT OF THE BUILDING OF NEW SUSTAINABLE NEIGHBORHOODS: THE CASE OF SAN CAYETANO IN MEJÍA, QUITO METROPOLITAN AREA, ECUADOR}

Susana Moreno Soriano², Francisco Javier González González³, Mireya Gutiérrez García ${ }^{4}$ Erika Raquel Ramos Fagundez ${ }^{5}$, María de Lourdes Delgado Posada ${ }^{6}$, Isabella Ribadeneira Moncayo ${ }^{7}$

\section{RESUMEN}

En este artículo se analiza el proceso de participación-involucración llevado a cabo por una comunidad de propietarios de suelo en Ecuador, en el contexto de construcción de un nuevo barrio. En el proceso, cuyo hilo conductor es la sustentabilidad, conviven la empresa privada (que promueve la iniciativa) y las políticas públicas que se orientan a facilitar el acceso a la vivienda. La investigación evidencia la presencia de una oportunidad de incorporar a la empresa privada en la generación de las comunidades locales, más allá de la mera producción del espacio urbano. Asimismo, el estudio revela que los vecinos tienen dificultades para pasar de la autoconstrucción y la informalidad a procedimientos formales. Finalmente, queda de manifiesto la potencialidad de integrar la autoconstrucción dentro del esquema de negocio de la empresa.

Palabras clave: desarrollo sustentable, barrios, vivienda económica, empresas, autoconstrucción.

\section{ABSTRACT}

\begin{abstract}
This article analyzes the participatory-engagement process carried out with a community of owners of land in San Cayetano, Mejía, Ecuador in the context of the construction of a new neighborhood. The process, whose unifying thread is sustainability, involves the coexistence of a private company that promotes the initiative, and the public policies that facilitate access to housing. The research demonstrates that there is an opportunity to incorporate private companies into the creation of local communities, beyond pure urban development. Likewise, the study reveals that the neighbors have difficulty transitioning from self-building and informality to formal procedures. Lastly, the potential for integrating selfbuilding into the company's business model is apparent.
\end{abstract}

Keywords: sustainable development, neighborhoods, subsidized housing, companies, self-building.

Artículo recibido el 21 de marzo de 2016, aceptado el 31 de mayo de 2016

\footnotetext{
[1] Artículo basado en los resultados de una investigación patrocinada por la Cátedra Barrios Sostenibles Maktrade Corp. S.A Universidad Europea de Madrid, y titulada "Elaboración de una metodología para intervenciones urbanas sostenibles a escala de barrio, con microfinanciación", CAT001501.

[2] Departamento Tecnología y Gestión de la Edificación, Universidad Europea de Madrid, España. susana.moreno@universidadeuropea.es [3] Departamento de Urbanismo, Civil y Aeroespacial, Universidad Europea de Madrid, España. franciscojavier.gonzalez@universidadeuropea.es

[4] Departamento Tecnología y Gestión de la Edificación, Universidad Europea de Madrid, España. mireyagutierrezg@gmail.com

[5] Departamento Tecnología y Gestión de la Edificación, Universidad Europea de Madrid, España. erika_rrf@hotmail.es

[6] Departamento Tecnología y Gestión de la Edificación, Universidad Europea de Madrid, España. I.delgado.posada@gmail.com

[7] Departamento Tecnología y Gestión de la Edificación, Universidad Europea de Madrid, España. isariba2000@gmail.com
} 


\section{INTRODUCCIÓN}

El diseño urbano, a escala intermedia, es un fructífero campo para la aplicación de criterios de desarrollo sustentable. Su escala hace posible analizar espacial y funcionalmente los flujos de energía y materiales como también evaluar su metabolismo urbano, manteniendo la eficiencia y funcionalidad de los sistemas. La calidad de vida urbana y la cohesión social del vecindario se constituyen, desde este enfoque, en elementos clave a considerar junto a la calidad del soporte físico. Las estrategias sobre la energía, el uso sustentable del agua, el tratamiento de los residuos y el uso de los materiales; así como la movilidad interior y la presencia de elementos naturales, tienen como objetivo que el metabolismo de los nuevo asentamientos produzca una huella ecológica que no consuma la capacidad de carga del territorio (Rueda, 2012).

En esta transformación de los barrios hacia un modelo de sostenibilidad se requiere necesariamente de la involucración de sus habitantes actuales o futuros (Jacques, 2003; Pindado, 2000; Rebollo, 2003; Pöppinhaus, 2005; y Wheeler, 2004). En este artículo se concibe la participación como un proceso de diseño botom-up, es decir, una dinámica que incorpora las visiones de diferentes actores sobre problemas complejos, como el del hábitat, y que en diversas etapas de síntesis consigue llegar a soluciones complejas a través de consensos (Velázquez y Verdaguer, 2005).

El punto de partida de este tipo de propuestas consiste en llevar a cabo un diagnóstico compartido de las necesidades, trabajando con escenarios, ideas y aspiraciones de los participantes. Este diagnóstico participado es, pues, la primera etapa de síntesis de este proceso de "abajo hacia arriba". La necesidad de atender a unos puntos de partida asociados a aspectos concretos y a 
problemáticas concretas para los participantes, es una premisa detectada en casi todas las metodologías, y en algunas de ellas, como la European Assembly Scenario Workshop (2012), este elemento es el centro de la dinámica a poner en marcha. Simplificando, en esta fase se tratan temas clave por todos los actores y se construye un escenario por cada tema. Con la evolución de las reuniones, el aspecto negociador se enfatiza para llegar a una jerarquía de problemas y oportunidades asumida por todos los participantes.

Este método se puede implementar de diferentes formas, entre las cuales son especialmente interesantes aquellas que poseen un carácter lúdico ${ }^{8}$. En este sentido, como parte de una implementación lúdica y parcial del proceso, se ha mostrado muy útil el diseño colaborativo, que tiene una larga tradición teórica (Lerup, 1974; Valdés, Zen y Basterrechea, 2013), ya cuantiosas experiencias en Latinoamérica ${ }^{9}$ y es considerado como una aplicación concreta de lo que Tironi (2015) denomina coproducción, sin pre-concepciones y en plano de igualdad entre el vecino y el técnico.

Este punto de vista sobre los procesos participativos y las técnicas comentadas se ha vertido en el diseño de participación para la construcción de un barrio en Ecuador, tratado como una investigación formal, que a continuación se presenta.

\section{EL CONTEXTO DE LA INVESTIGACIÓN: UN PROYECTO DE BARRIO SUSTENTABLE EN SAN CAYETANO}

La investigación se realiza sobre un proceso participativo llevado a cabo en una comunidad que se está construyendo en el barrio de San Cayetano, en el Cantón Mejía, limítrofe por el sur con el Cantón de Quito, en Ecuador.

San Cayetano ocupa aproximadamente 18 ha y se encuentra al sur de la parroquia de Santo Domingo, en el Cantón Mejía. Se trata de un territorio en el que predomina el uso agrícola. Sin embargo, su proximidad a Quito por el sur, señala para esta área una oportunidad conectada al futuro desarrollo urbano del Plan Masa INIAP Santa Catalina. Cuando se dio inicio a este estudio el barrio contaba con un plan parcial ya desplegado con la urbanización del sistema viario y los servicios básicos, y 172 de alrededor de un total de 500 lotes ya habían sido escriturados.

La empresa privada a cargo de dicha urbanización y la cual ha realizado la venta de lotes utilizando el microcrédito como herramienta económica, pretendió dar un paso más e incorporar procesos sustentables a las próximas etapas de construcción del barrio. Para ello se propuso un proyecto de investigación amplio que

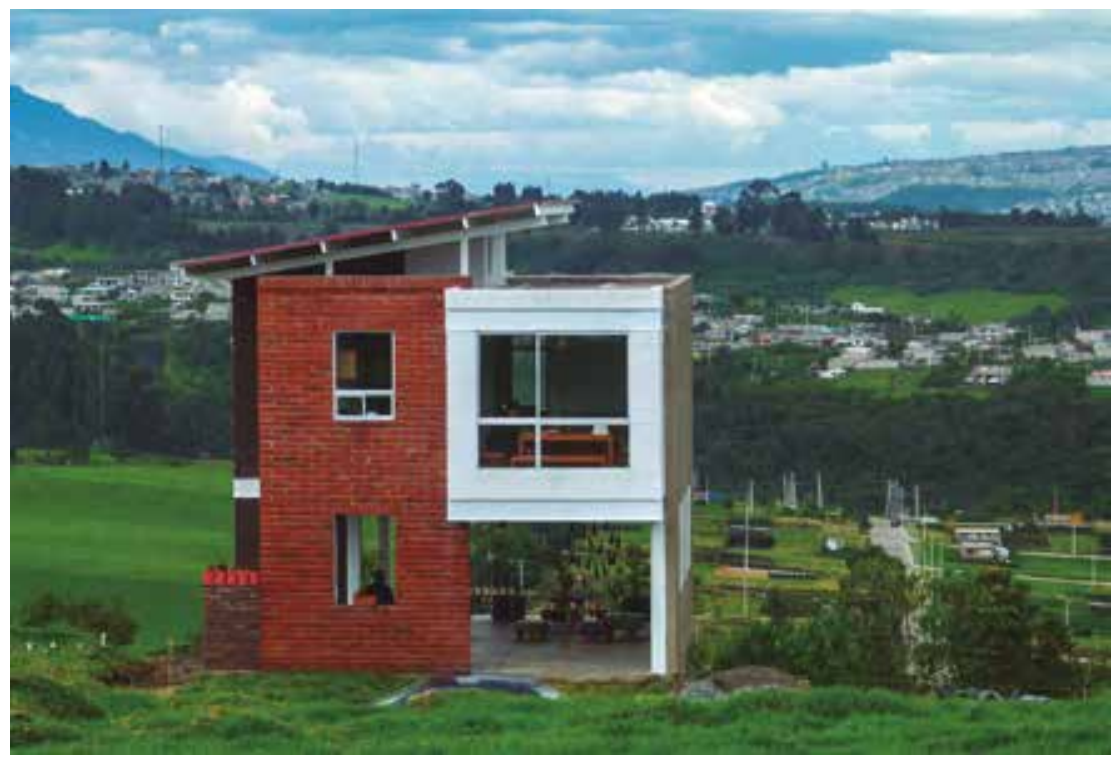

Figura 1 San Cayetano al fondo con casa modelo en primer plano. Fuente: Fotografía de Isabella Ribadeneira

[8] A modo de ejemplo, valen las experiencias de algunos grupos de activistas urbanos en España. Paisajes transversales inventaron un "Trivial Pursuit" callejero en el barrio de Begoña, en Madrid, para que la gente, a través de este juego de preguntas y respuestas aportara conocimiento sobre el barrio, sobre el estado percibido de los espacios públicos, pero en correlación con otras muchas preocupaciones. Véase: http://www.paisajetransversal.org/

[9] Como referencia interesante, pero ni mucho menos única, sirva los procesos participados para diseñar las UVA (Unidades de Vida Articulada), equipamientos barriales de la ciudad de Medellín. Véase: http.//www.edu.gov.co/site/proyectos/unidades-de-vida 
testeara estrategias para llevar al barrio a un funcionamiento de consumos de carbono muy bajos y mantener la eficiencia y funcionalidad de los sistemas. Como parte de este proyecto macro, se plantea una investigación parcial relativa al proceso de participación, objeto de este artículo.

El modelo de negocio está basado en la construcción a gran escala de vivienda social con financiación pública para quintiles sociales entre el 1 y el 2. La necesidad de alojamiento de estos grupos sociales, supone entender la vivienda como un espacio de reproducción familiar, en un sentido extenso. Esto significa que la vivienda es la sede de crecimiento natural no ya de una familia, sino de varios núcleos familiares, con lo que la flexibilidad en el crecimiento del espacio habitable se convierte en un factor fundamental. Por otro lado, la vivienda se considera también un espacio de producción doméstica, con estancias reservadas para el comercio o la manufactura como parte de las actividades que traen ingresos a las familias. Desde el punto de vista de la ordenación del conjunto, el modelo inmobiliario pretende aumentar la calidad urbana con la presencia de servicios urbanos bien articulados, a partir de desarrollos de gran densidad en los que la mezcla tipológica se encuentre reducida a viviendas individuales de no más de dos plantas.

\section{OBJETIVOS Y ALCANCE}

Concretamente, el objetivo del artículo es relatar una experiencia de participación promovida desde la empresa privada, en el contexto de la construcción de un barrio sostenible y en el área metropolitana de Quito. Ligado a un proyecto urbanístico y en especial a sus viviendas, se pone en marcha un proyecto de investigación que pretende explorar qué criterios de sustentabilidad pueden ser aplicados a la actuación.

El proceso de participación es diseñado como un apartado especial de dicha investigación y pretende explorar el tipo de reacción de los intervinientes sobre dos aspectos: a) lo relativo a la sustentabilidad, a escala de barrio y de edificio, y b) el papel de la empresa privada, promotora del barrio, como impulsora de la participación, rol normalmente reservado a las entidades públicas locales.

\section{METODOLOGÍA}

La investigación se ha diseñado en tres fases y para cada una de ellas se ha desarrollado un método de interacción con el vecino-cliente. Estas fases son:

\section{Información-vinculación}

Esta etapa no sólo es la inicial, sino que supone un momento delicado, en el que se validan las posibilidades de ocupar un papel en el proceso de construcción de la comunidad del barrio por parte de la empresa. La capaci- dad de congregación de los, hasta ahora clientes, ha de desembocar en un tipo de relación más parecida a la de un facilitador de los procesos dentro de una comunidad, papel habitualmente llevado a cabo en el entorno de lo público.

Para ello, el trabajo en asambleas informativas sobre los objetivos perseguidos, las encuestas en las que se tratan temas relacionados con las condiciones de vida cotidianas, hábitos ecológicos, trabajo colaborativo y economía familiar, así como algunas entrevistas personalizadas, permiten ir identificando de forma provisional demandas compartidas y sujetos activos, de cara a la futura comunidad, que puedan cumplir un buen papel de interlocutores.

\section{Co-diseño}

Se pone en marcha un servicio de acompañamiento de familias con el fin de asesorarles mediante un técnico arquitecto o ingeniero acerca de cómo puede ser la casa que están pensando autoconstruir o que simplemente les gustaría tener. Para ello se pretende experimentar, dentro de las posibilidades normativas del planeamiento aprobado, diversas distribuciones con maquetas $2 \mathrm{D}$ de papel y 3D, lo que hace posible visualizar los deseos de la familia y encontrar las limitaciones espaciales de los mismos. Asimismo, se asesora en relación a la organización de los espacios dentro de una vivienda: las posibilidades de ampliación y su funcionamiento cotidiano. En este momento se busca explorar la dimensión espacial de los datos surgidos de la encuesta de la fase anterior.

\section{Talleres de vivienda y barrio}

Ideados como espacio de encuentro entre la empresa y los futuros vecinos, se proponen dinámicas de diagnóstico colectivo de las necesidades. En principio, son pensadas para la definición de tipos de vivienda pero, casi inmediatamente, se modifica el método, ampliándolo, para entender cuáles son las demandas sobre el futuro barrio. Se aborda la participación de un modo sectorial: se testan los temas relativos las necesidades de habitabilidad para pasar a los relativos al metabolismo urbano (al uso de la energía, el agua y los residuos sólidos urbanos), así como a los problemas de movilidad y de transporte y conexión con el resto de la ciudad. La autoconstrucción y sus problemas técnicos y económicos, constituye otro tema que se incorpora en la discusión. El número de reuniones se considera que ha de ser abierto en función de la evolución del proceso.

\section{EL CASO DE ESTUDIO: FASES Y RESULTADOS OBTENIDOS} EN EL PROCESO DE PARTICIPACIÓN

El proceso fue trazado en sus líneas maestras por la Cátedra Barrios Sostenibles en Madrid. Por parte de la empresa, se creó una división dedicada exclusivamente a hacerse cargo de los procesos de participación, inte- 


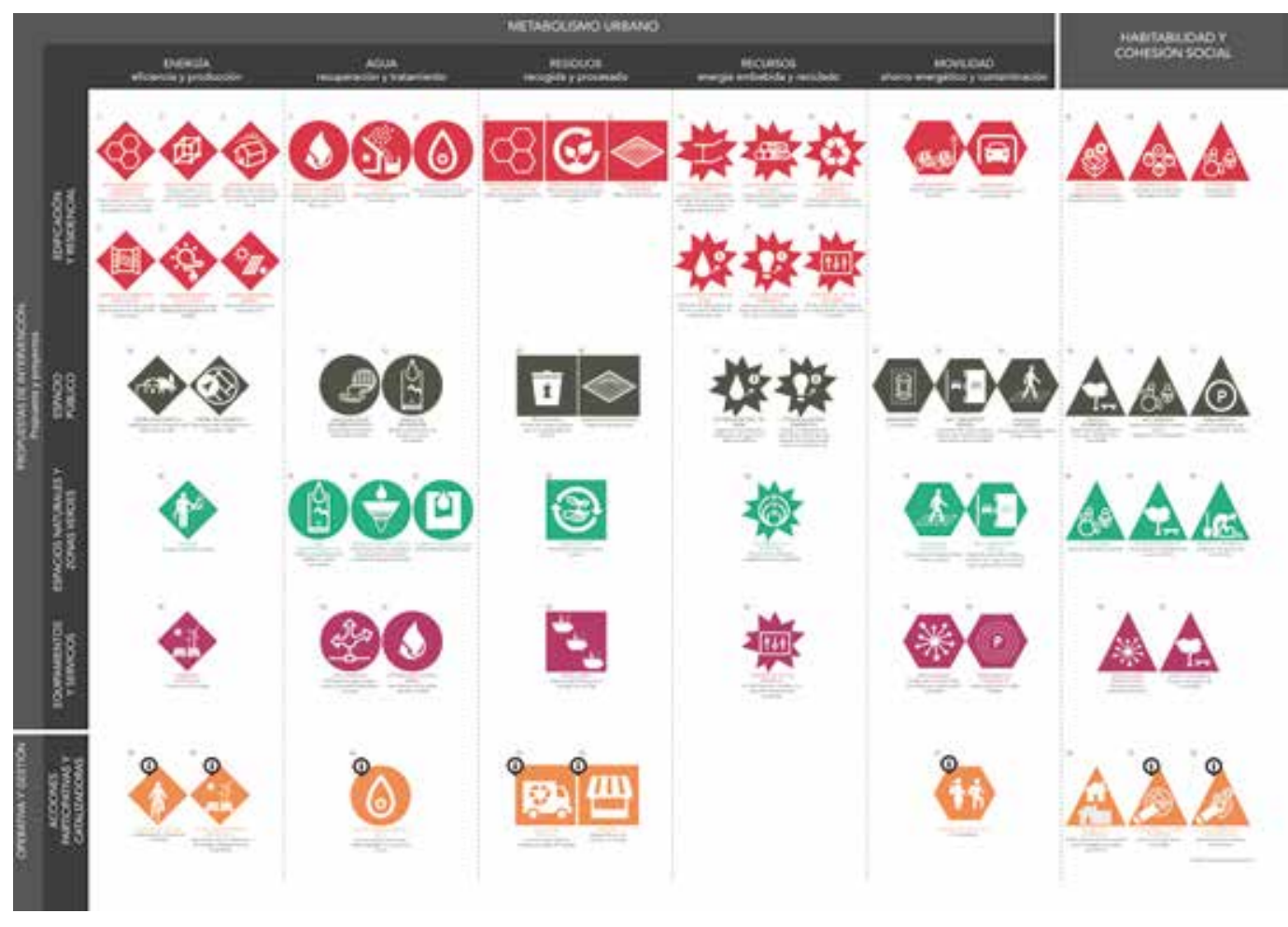

Figura 2 Matriz de las estrategias de diseño urbano sustentable. Fuente: Elaboración del autor.

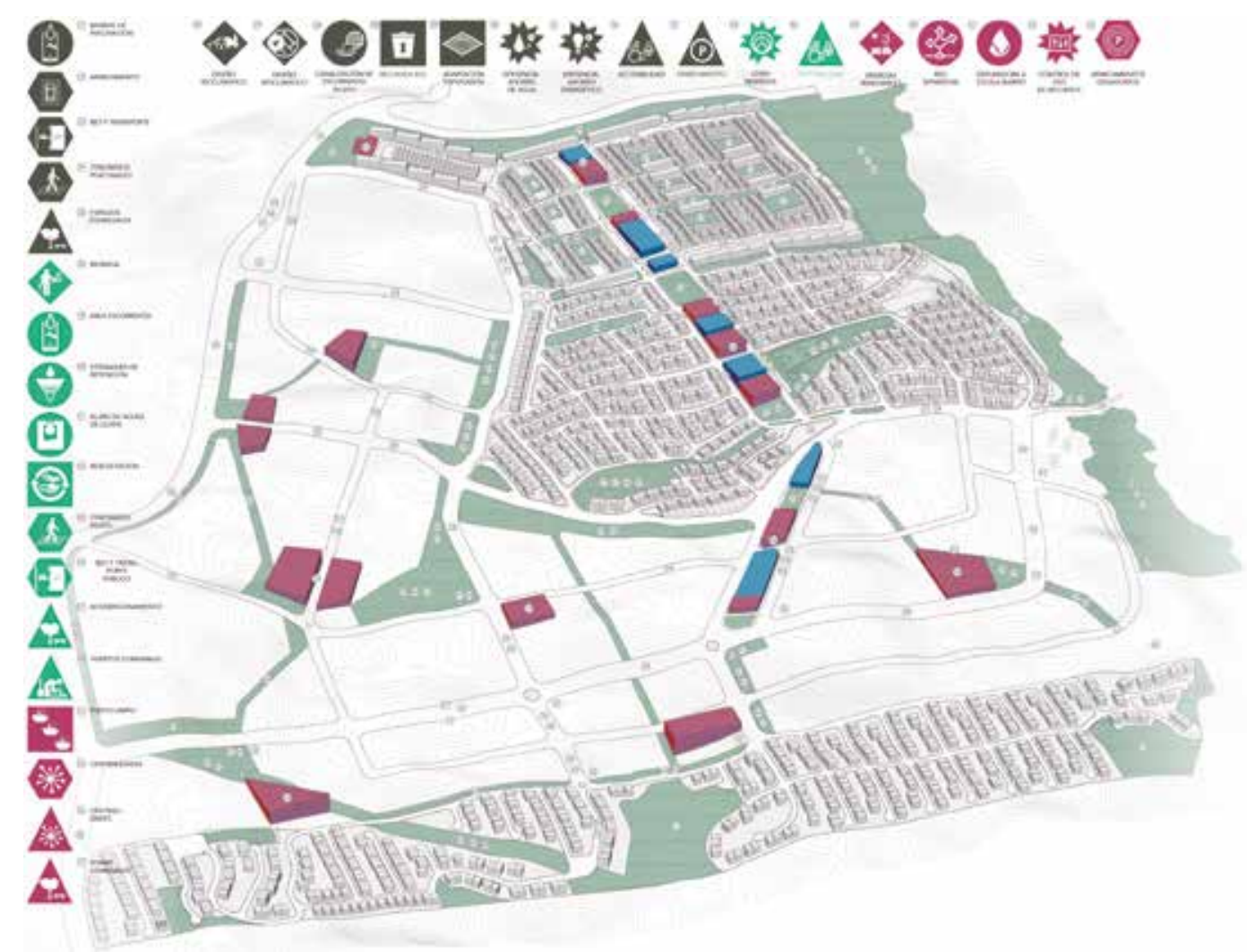

Figura 3 Propuesta de diseño urbano sustentable para San Cayetano-El Belén. Fuente: Elaboración del autor 
grada por un gestor y dos arquitectos encargados de convocar y dirigir dichas causas, así como de canalizar el asesoramiento de la Cátedra. Además de esto, se contó con el asesoramiento especializado de varias personas de otros departamentos técnicos de la empresa. Para llevar a cabo los citados procesos se dispuso de una pequeña construcción tradicional existente en la zona, que se identificó como un lugar significativo para la comunidad y que se rehabilitó para servir de sede.

Este recinto, que funcionó como casa comunal, tiene una dotación básica para realizar reuniones de pequeño formato con alrededor de veinte participantes. Teniendo en cuenta que tomaban parte en las reuniones varios miembros de cada familia, se trabajaba, por un lado, con los adultos y se planificaban actividades en paralelo con los niños. El calendario de los procesos comenzó en mayo de 2015 y se prolongó durante siete meses. Las reuniones se efectuaron durante el fin de semana, momento en el que también otras familias aprovechaban para recorrer el lugar y cuidar de sus lotes o de sus huertos. Se completaron, así, las tres primeras etapas previstas en la planificación, es decir, de informacióninvolucración, de co-diseño y el taller de vivienda; fases que se analizan en lo que sigue.

Siguiendo la metodología indicada, se da relación de algunos aspectos organizativos y los resultados obtenidos en cada etapa:

\section{Proceso de Información-Involucración}

Se parte de una condición de confianza mutua que se funda en la buena relación ya establecida anteriormente entre empresa y cada uno de los participantes, pero, a su vez, la doble condición de clientes y vecinos requiere establecer pautas claras que ayuden a redefinir el papel de la empresa. A efectos prácticos, se cuenta con la fuerza de ventas de la empresa ya que son los agentes que han ido generando confianza con los clientes a los que se han vendido los lotes del barrio. Para los vecinos estas son las personas de referencia en el proceso de financiación mediante microcrédito. El punto de transición entre lo que es formar parte de una red clientelar a constituirse en una comunidad de barrio, comienza con la referida encuesta. Esta se realizó telefónicamente como medio no sólo de informar del proceso y de recopilar información objetiva, sino también para comprobar la disponibilidad de los clientes para constituirse en comunidad. Inicialmente, la intención de adherirse al proceso fue positiva en el $87 \%$ de los casos.

\section{Co-diseño}

La idea directriz fue la de pensar de modo colaborativo -la familia y los técnicos- cómo organizar los espacios de la vivienda conforme a las necesidades originales, cómo deducir otras que surjan en el proceso y cómo incorpo- rar los temas de sostenibilidad y metabolismo urbano. Se trabajó, de este modo, con una maqueta de papel 2D, con habitaciones en papel en formas y colores distintos para confeccionar el puzle. Inmediatamente se pasa a una maqueta 3D con mobiliario a escala, ya que algunas personas no son capaces de imaginar los espacios. Se observa que las posibilidades de distribución de un programa son limitadas y toma protagonismo entonces la idea de trabajar sobre el co-diseño de la fachada como medio de alcanzar una imagen organizada del conjunto del barrio y, a la vez, permitir la personalización de cada casa, que era un aspecto muy importante para todos los participantes.

\section{Talleres de vivienda y barrio}

Ya que la dinámica de los talleres pretende realizar el diagnóstico colectivo de los problemas de alojamiento de los asistentes, las reuniones se dedican a un tema inicial, que en el transcurso de la reunión se entremezcla con otros, lo que permite establecer correlaciones de interés de los vecinos. Los temas iniciales que se abordaron se agruparon en cuatro bloques: necesidades de espacio previstas (con base en las experiencias de co-diseño previas), hábitos cotidianos ecológicos (agua, residuos, confort), condiciones económicas de las familias y barreras para acceder a una vivienda, y conocimientos para autoconstruir. En un momento determinado se detecta que el interés se desplaza hacia la necesidad de espacios para la comunidad en el barrio y el modo de incorporar "la minga"10 como método de trabajo colectivo de transformación de los espacios colectivos. Las reuniones comienzan con un resumen de lo tratado en las anteriores. En cuanto a la capacidad de la empresa de ir generando comunidad, se observa la presencia continua y sistemática de las personas más interesadas, un grupo total de unas 30 familias, y nuevas incorporaciones fluctuantes, sin que se detecte un patrón claro de crecimiento de este tipo de afluencia, mostrándose más bien aleatorio.

En el transcurso de las reuniones, se establecieron prioridades como la autoconstrucción y la construcción por etapas, ya que muchas personas tienen habilidades para construir por sí mismos sus viviendas y desean controlar y participar personalmente en el proceso de construcción. También se demostró que la mayoría de las personas quiere comenzar con un tipo de vivienda básica de dos habitaciones, un baño, cocina-comedor y sala en planta alta y había mucho interés por disponer de una planta baja diáfana para instalar un negocio o alquilar un departamento.

El argumento económico se desveló, en efecto, como crucial para las familias. Se subrayó como prioritario resolver a través de un acuerdo el problema económico que comparte la mayor parte de las personas que vive actualmente en vivienda arrendada; el cual genera que muchas de ellas deseen trasladarse al barrio levantando una construcción a media agua que no requeriría permiso ${ }^{11}$. Este es un argumento que dificulta superar los 


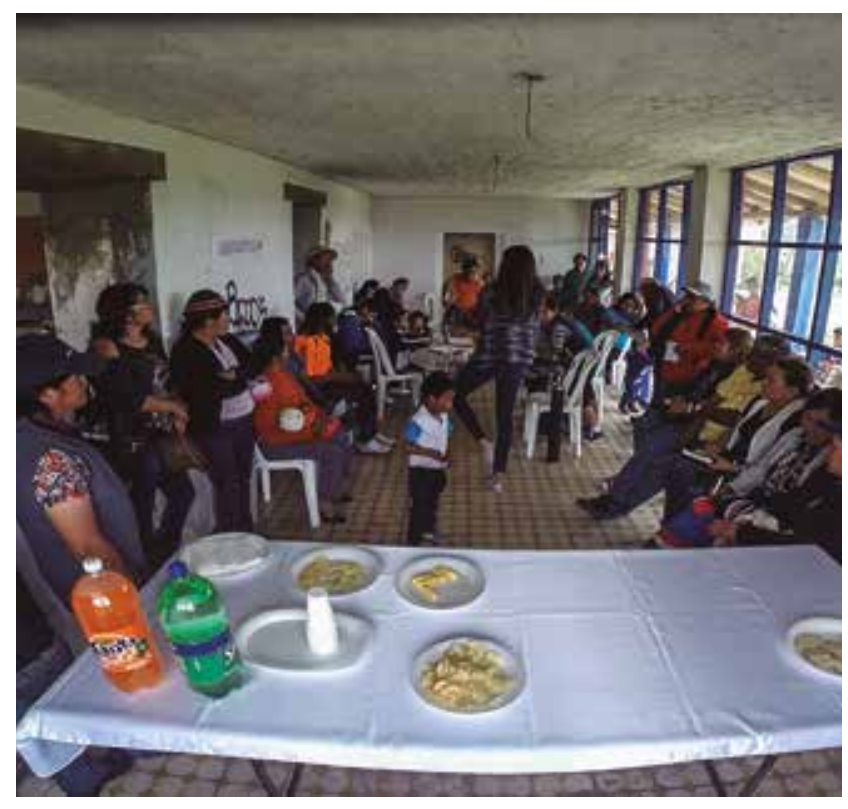

Figura 4 Una de las reuniones celebradas. Fuente: Fotografía de Carmen Elena Ribadeneira.

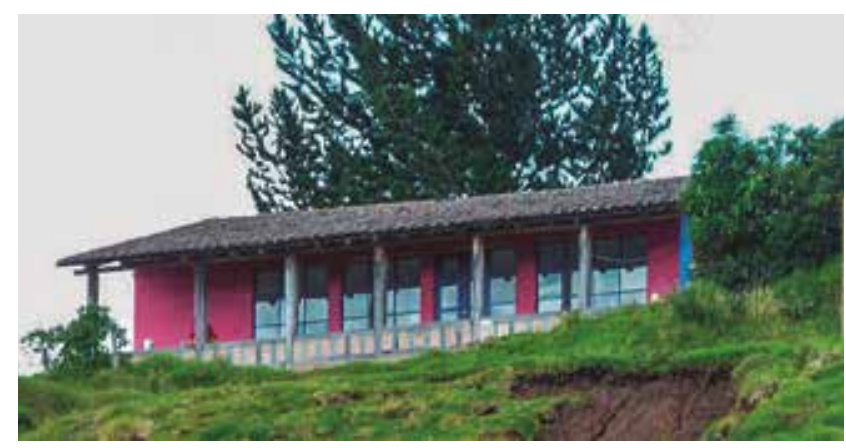

Figura 5 La casa comunal. Fuente: Elaboración del autor.

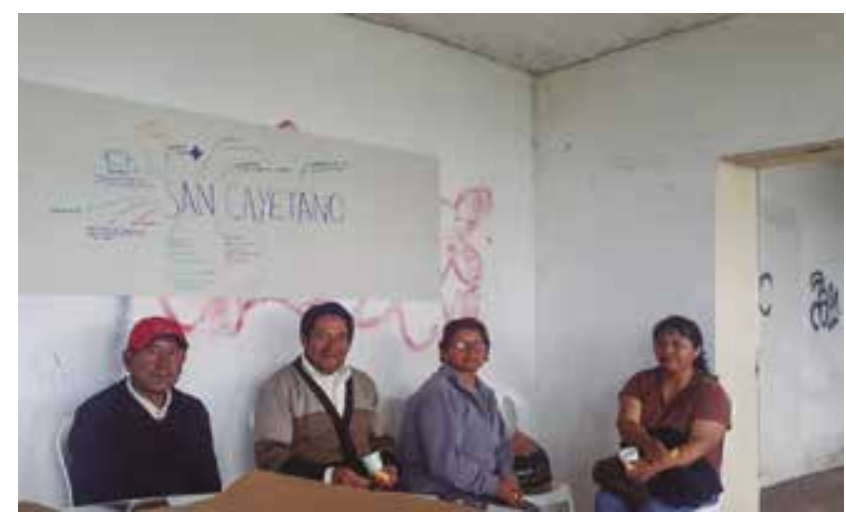

Figura 6 Vecinos participantes en una reunión. Arriba esquemas elaborados durante el proceso. Fuente: Elaboración de David Padilla. 


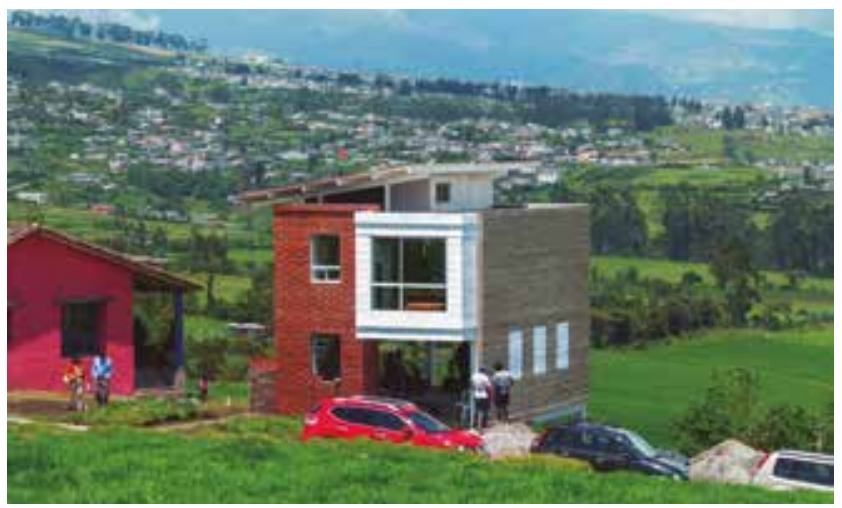

Figura 7 La casa modelo se edificó junto a la casa comunal. Fuente: Fotografía de Carmen Elena Ribadeneira.

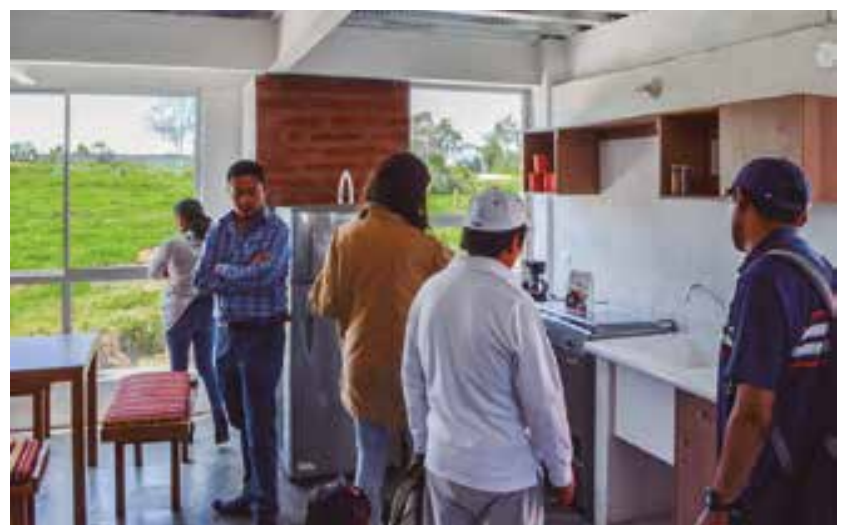

Figura 8 Una visita de varios vecinos acompañados de Isabella Ribadeneira a la vivienda situada en la planta alta de la casa modelo. Fuente: Elaboración de David Padilla.

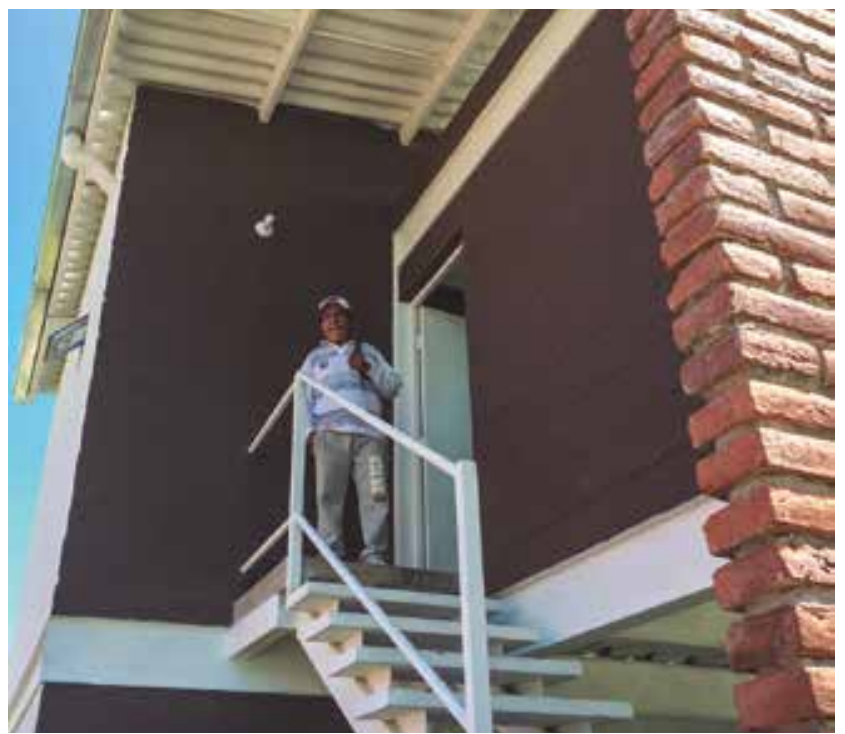

Figura 9 Un vecino en lo alto de la escalera exterior de acceso a la vivienda. Fuente: Elaboración de Isabella Ribadeneira. 


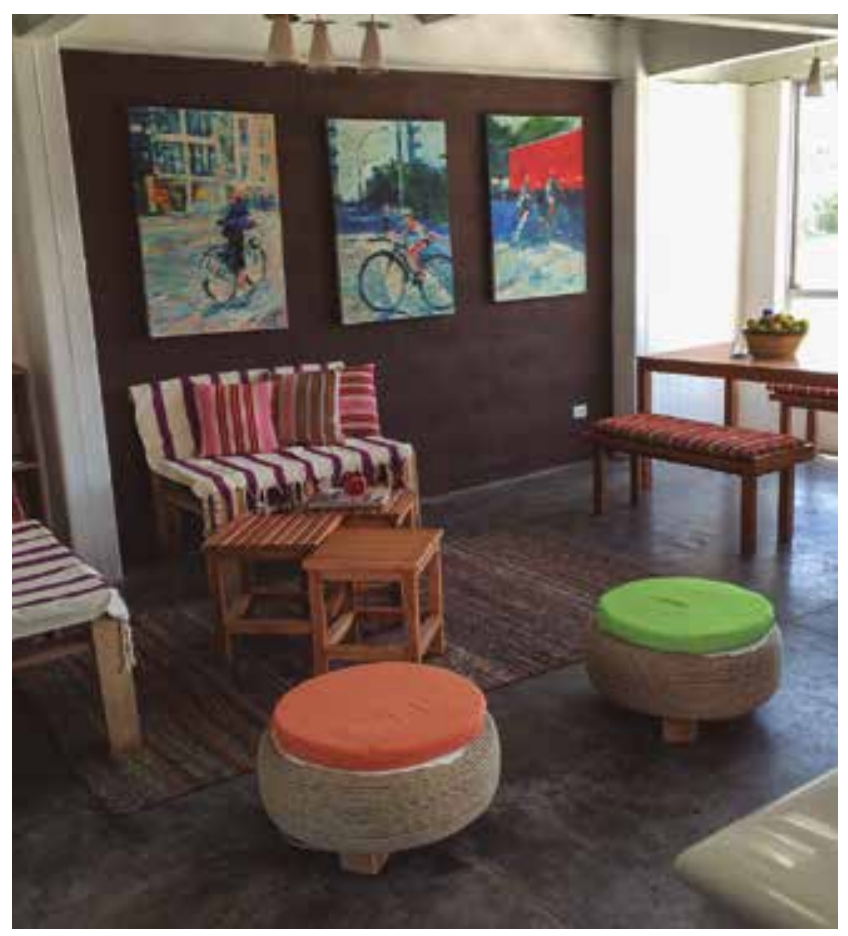

Figura 10 Vivienda en planta alta. Fuente: Isabella Ribadeneira

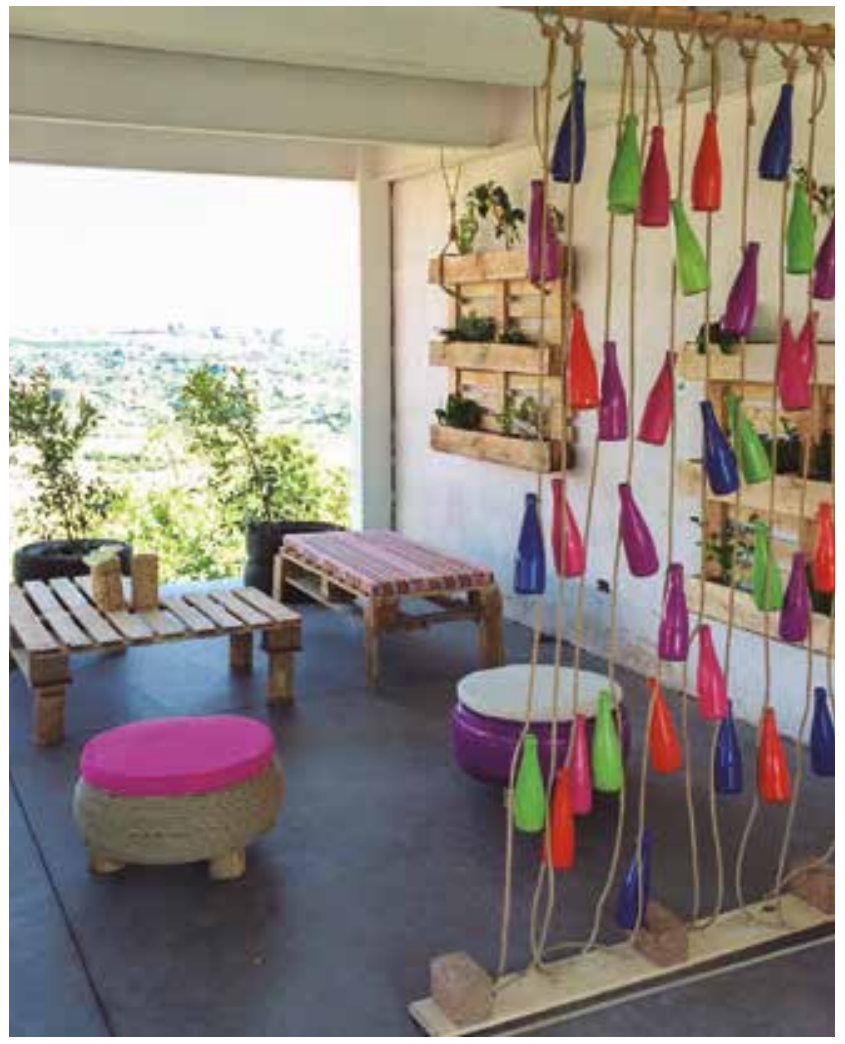

Figura 11 Planta baja disponible para usos futuros, alternativos al de vivienda. Las piezas de mobiliario se diseñaron de acuerdo a criterios de buenas prácticas de reutilización de materiales. Fuente: David Padilla 

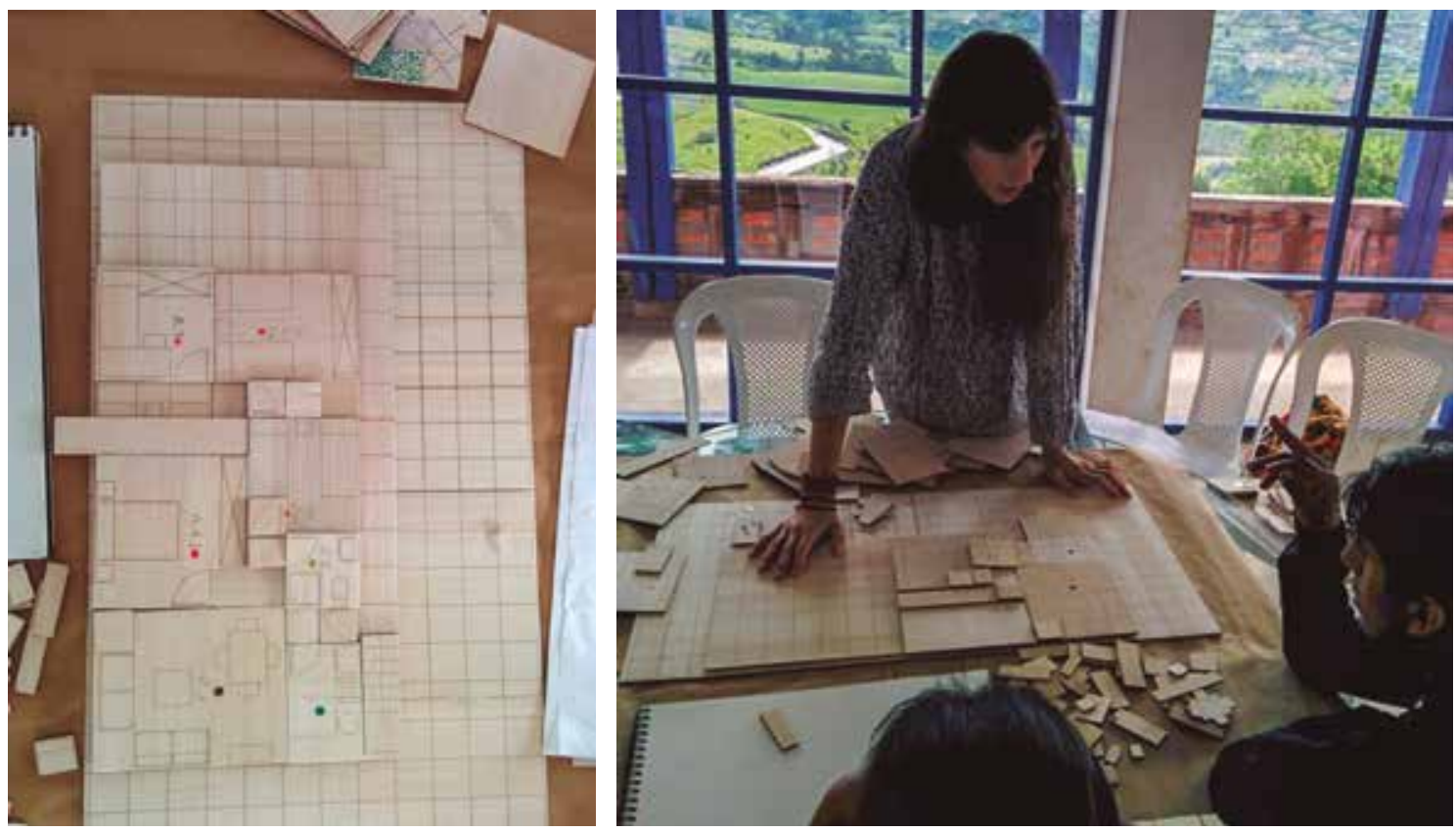

Figura 12 Plantillas elaboradas para el co-diseño. Fuente: Elaboración de Esteban Naranjo.

modelos de autoconstrucción informal. Si bien la aceptación de una vivienda proyectada, fruto de un proceso formal, se ha dado mayoritaria en el papel, el miedo de las familias a la inestabilidad económica a mediano plazo potencia soluciones de autoconstrucción, aun cuando éstas son conscientes de la menor habitabilidad de tales viviendas.

El análisis de los resultados de las primeras reuniones lleva al equipo a proponer la construcción e inauguración de una casa modelo ante la necesidad de impulsar el proceso resolviendo las inquietudes y las dudas detectadas por los participantes. Aprovechando lo aprendido en la fase de co-diseño, este prototipo ha evolucionado en los procesos a partir de un anteproyecto con una idea fuerte de vivienda progresiva con crecimiento hacia abajo y hacia el fondo del lote. Es decir, una forma de asegurar una construcción organizada y de calidad, y, asimismo, una estrategia de control de costes basada en una casa mínima en planta alta ${ }^{12}$.

A partir de la cuarta reunión se comenzó a trabajar en el taller de vivienda sobre el modelo de casa con crecimiento hacia abajo. Este taller se extendió durante el periodo de construcción de la casa, que tuvo una duración de dos meses. Dicha construcción se emplazó junto a la casa comunal donde se celebraban las reuniones, lo que fue clave para facilitar la sintonía entre la empresa y los vecinos ya que facilitó la explicación por los expertos de conceptos previamente planteados, relacionados con el diseño abierto, la construcción por fases y los elementos de sustentabilidad y adaptabilidad que se estaban discutiendo. También permitieron sacar a la luz las limitaciones de los vecinos para asumir el modelo de viabilidad económica propuesto por la empresa. La vivienda se inauguró el 7 de noviembre de 2015 y, a continuación, se celebraron dos reuniones más, pero a partir de este momento, se trabajó especialmente con las personas que acudieron a conocer la casa o con familias propietarias de lotes que siguieron acudiendo a disfrutar del lugar los fines de semana. La construcción de esta casa prototipo supuso un hito del proceso, que sirvió para testar las opiniones de los participantes y de nuevos vecinos, quienes, desde entonces, han mostrado notorio interés ${ }^{13}$. Gracias a la construcción de una casa modelo fue posible enfrentar la preocupación de los vecinos por algunos temas que no surgieron en las reuniones iniciales, como las restricciones de las ordenanzas en cuanto a retiros, alturas y crecimiento hacia arriba, que es la forma de construcción de vivienda predominante en el país. cien personas. El primer momento de afluencia fue a la inauguración. Se pensaba haber hecho un gran evento pero finalmente se hizo algo más pequeño. Se invitó a unas cuarenta familias que ya tenían escrituras. Entonces se vio que las personas que habían estado en los procesos sentían la casa como suya y explicaban a los otros todos los detalles de cómo se había hecho. Después contactamos con muchas personas, hubo un momento en que las personas no acudían a reuniones sino que nosotros íbamos los fines de semana y hablábamos con los propietarios que iban a San Cayetano a cuidar de sus lotes". 
En cuanto a las prioridades para el barrio se llegó a un acuerdo notable en cuanto a las necesidades compartidas y las formas de lograr abordar algunas de ellas. Entre ellas se partió de necesidades y preocupaciones básicas que se consideraban la prioridad en las primeras reuniones, como la seguridad del barrio y la importancia de contar con un centro de salud y una guardería. Se continuó con la idea de que es necesario un proyecto común de barrio y se plantearon propuestas de organización de mingas preparar dotaciones para el barrio: Canchas deportivas, senderos y amueblamiento de los espacios públicos, para finalmente, compartir ideas sobre una visión de barrio sustentable con oportunidades económicas para sus habitantes. Las cualidades naturales del barrio y el área natural protegida se reconocieron como un elemento de valor para la propia comunidad. Se entendió que las habilidades y el interés por los huertos que varios de los propietarios tenían, permitirían abordar proyectos en común como organizar mercados para vender productos de elaboración propia y atraer una cierta actividad turística basada en estos productos artesanales o ecológicos.

\section{DISCUSIÓN}

En lo que se refiere a la capacidad de generar comunidad y una red con capital social duradero, se entiende que la experiencia aquí expuesta avala la capacidad de la empresa para hacer de facilitador. Sin embargo, es importante aclarar algunas ideas para diseñar mejor la información inicial a los primero clientes y luego vecinos. Los vecinos-clientes se dirigen a la empresa como si del propio ayuntamiento se tratara, con peticiones sobre la necesidad de acondicionamiento de espacios verdes, deportivos, de juego, de lugares comunitarios donde reunirse o espacios comerciales que permitan una progresiva apropiación tanto de los lotes privados como de dichos lugares públicos que todavía no están preparados para ser usados de un modo satisfactorio. Las reclamaciones no tienen carácter de quejas en el contexto empresa-cliente, sino que se producen a medio camino entre la petición de mediación ante los poderes públicos y una propuesta de autogestión apoyada desde la empresa.

En lo que se refiere a la experiencia de co-diseño, la metodología llevada a cabo demuestra un alto grado intercambio entre expertos y no expertos. Los ejercicios de co-diseño (Tironi, 2015), consistentes en diversas actividades con plantillas para el diseño, demostraron ser adecuados pero poco operativos para permitir diseños personalizados. No obstante, estas actividades permitieron comprender otras formas de personalizar el diseño que eran demandadas por los vecinos.

El método de diagnóstico colaborativo demostró ser adecuado durante los talleres de vivienda. La organización de sesiones monográficas hizo aflorar otros temas quizá más urgentes para los participantes. El proceso facilitó que los vecinos, además de propuestas, plantearan vías de implementación. Se destacó la necesidad del acercamiento a la administración con iniciativas concretas y se encontró disposición a colaborar en las soluciones mediante trabajo colaborativo en "mingas".
La construcción del prototipo, su inauguración y la posibilidad de experimentar el espacio construido supuso un momento de cierta catarsis sobre los debates previos. Muchas dudas se disiparon y han permitido al equipo investigador entender cómo mejorar la presentación de algunos temas en posteriores procesos, rebajando el grado de abstracción inicial y reforzando el trabajo previo con maquetas. Un dato significativo es que se logró una aceptación muy alta por parte de los vecinos de la normativa de municipio referente a ocupación, retiros y altura edificada, lo que se valoró como vía para asegurar un resultado final coherente, algo que al principio se percibía como una imposición poco aceptable. El modelo de casa con crecimiento progresivo hacia abajo, alcanzó un grado de aceptación muy alto.

Otro aspecto interesante de la experiencia reside en cómo los temas relacionados con lo urbano aparecen en el proceso de participación de forma no prevista. Las preocupaciones de los vecinos se extienden desde el hábitat básico, la vivienda, hacia un contexto mayor, el hábitat urbano, ya que las necesidades colectivas surgen casi a la vez que las necesidades de alojamiento más primarias. 


\section{CONCLUSIONES}

A partir de esta discusión se han deducido dos conclusiones básicas:

1. Existe un nicho disponible para la incorporación de una empresa privada en la generación de las comunidades locales, más allá de la mera producción del espacio urbano. La experiencia demuestra que el apoyo con microcréditos y la confianza generada en la venta de lotes con títulos de propiedad asegurados y un nivel de calidad en la urbanización, no habitual en este tipo de procesos de urbanización, permiten una situación de autoridad reconocida que lleva a que los nuevos vecinos traten a la empresa como interlocutor con la administración en la dinámica colectiva que supone generar una nueva comunidad. En este sentido, la estrategia seguida de organizar un proceso de participación-involucración supone construir una plataforma para que los futuros vecinos vayan generando su capital social poco a poco, esto es, conformar sus redes de apoyo mutuo en el barrio a partir de la red de contactos de la propia empresa.

Las ventajas del procedimiento son múltiples. Por un lado, en lo que se refiere a los intereses empresariales, cuanto más crezca la comunidad, cuanto mejor sea la calidad física del barrio, mayor capacidad de atracción tiene el barrio y, por tanto, más oportunidades de venta de lotes o viviendas. El interés de potenciar el capital social de la comunidad, incluso utilizando los recursos propios de la empresa, permite reforzar un papel de intermediación entre vecinos y la administración local, ganando una confianza que revierte en su prestigio empresarial.

Por otro lado, los vecinos disponen del apoyo de la empresa para canalizar los recursos de fuerza de trabajo y así mejorar la calidad física del barrio allí, donde el ayuntamiento no llega con sus propios recursos y la empresa, de un modo formal, ya ha cumplido su labor como urbanizador. La "minga" se puede beneficiar de la capacidad organizadora de la empresa.

Por último, el papel de intermediación de la empresa permite que la administración local deje espacio para los procesos auto-organizativos de los vecinos que suplan las dificultades de provisión de servicios que puedan tener el Ayuntamiento, o consigue que se catalice y acelere la construcción del barrio.

2. El proceso también ha revelado la potencialidad de incorporar la autoconstrucción dentro del esquema de negocio de la empresa, por ejemplo, reutilizando materiales de otras promociones o animando a los vecinos a que aporten sus propios materiales para que sean utilizados conforme a criterios de sustentabilidad ambiental. La labor de formación que esto supondría no se ha puesto en marcha aún pero es un camino de dar voz a los vecinos en las próximas etapas que se considera con amplia capacidad de desarrollo. 
JACQUES, Manuel. Modelo de participación por afección: un modelo para el desarrollo de la ciudadanía local. Polis [en línea], 2003, $n^{\circ}$ 5. [Consultado 06 marzo 2016] DOI: $10.4000 /$ polis.6921Polis

LERUP, Lars. Changing Roles in Environmental Design: The Designer as Co-Learner. Journal of Architectural Education, volumen XXVI, 1974, n4, pp. 100-109. (Traducción del autor).

PINDADO, Fernando. La participación ciudadana en la vida de las ciudades. Barcelona: Ediciones del Serbal, 2000.

PÖPPINHAUS, Hubertus. Participación ciudadana, ¿Quí fa la Ciutat? En: HERRERO, Luis Fco Participación ciudadana para el urbanismo del siglo XXI. Valencia: ICAROCTAV- COACV., 2005.

REBOLLO, Óscar. Bases político-metodológicas para la participación. En: ARENILLAS, Teresa (coord.). Ecología y ciudad. Raíces de nuestros males y modos de tratarlos. Madrid: FIM, El Viejo Topo, 2003, pp. 287-302.

RUEDA, Salvador. Libro verde de sostenibilidad urbana y local en la era de la información. Barcelona: Centro de Publicaciones Secretaría General Técnica MAGRAMA, 2012.

TIRONI, Manuel. Redefiniendo la participación ciudadana, redibujando lo ciudadano: El plan de participación ciudadana del PRES Constitución. Arquitecturas del Sur, 2009, no36, pp. $52-65$.

VALDÉS, Sylvia; ZEN, Marcela de y BASTERRECHEA, Lucía. Diseño participativo y sustentable. Buenos Aires: Ediciones del CCC- Centro Cultural de la Cooperación Floreal Gorini, 2013.

VELÁZQUEZ, Isabela. y VERDAGUER, Carlos. Instrumentos para la intervención social activa: Talleres de futuro EASW en el urbanismo participativo. En: HERRERO, Luis Francisco (ed.). Participación ciudadana para el urbanismo del siglo XXI. Valencia: ICARO-CTAV- COACV, 2005.

WHEELER, Stephen M. Planning for Sustainability. Creating Livable, Equitable and Ecological Communities, $2^{\text {a }}$ Edición. Londres: Routledge, 2004. 